\title{
Lensless Imaging of Nano- and Meso-Scale Dynamics with X-rays
}

Jesse N. Clark ${ }^{1,2}$, Mariano Trigo ${ }^{3}$,Tom Henighan ${ }^{1}$, Ross Harder ${ }^{4}$, Brian Abbey ${ }^{5}$, Tetsuo Katayama ${ }^{6}$, Mike Kozina ${ }^{1}$, Eric Dufresne ${ }^{4}$, Haidan Wen ${ }^{4}$, Donald Walko ${ }^{4}$, Yuelin $\mathrm{Li}^{4}$, Xiaojing Huang ${ }^{7}$,Ian Robinson $^{8}$ and David Reis ${ }^{1}$

1. Stanford PULSE Institute, SLAC National Accelerator Laboratory, Menlo Park, USA

2. Center for Free-Electron Laser Science, Deutsches Elektronensynchrotron, Hamburg, Germany

3. SLAC, SLAC National Accelerator Laboratory, Menlo Park, USA

${ }^{4}$ Advanced Photon Source, Argonne National Laboratory, Argonne, USA

${ }^{5}$ Department of Physics, La Trobe University, Bundoora, Australia

${ }^{6}$ JASRI, Hyogo, Japan

${ }^{7}$ National Synchrotron Light Source II, Brookhaven National Laboratory, Upton, USA

${ }^{8}$ London Centre for Nanotechnology, University College London, London, United Kingdom

There is a fundamental interest in studying pico-second dynamics at the nano- and meso-scale in particles and structures as it can provide insight into their mechanical and thermal properties out of equilibrium and during phase transitions. Imaging over such short time-scales has been very challenging due to stringent temporal requirements. Here we report two types of lensless imaging experiments which exploit the coherence of modern x-ray sources to image dynamics at the nano- and meso-scale.

Lensless imaging relies on iterative phase retrieval to replace the role of a lens in a traditional imaging system [1]. Some of the advantages of this include greater sensitivity and removing some challenges with manufacturing high quality lenses. Using a spatially and temporally coherent source (such as xrays or electrons), diffraction patterns are collected from finite sized or extended objects. Iterative phase retrieval is then used to obtain a real-space image of the object by iteratively enforcing consistency with the measured data and real-space constraints.

Using the short pulses from an x-ray free-electron laser, we demonstrate three-dimensional imaging of the generation and subsequent evolution of coherent acoustic phonons on the picosecond time scale within single nanocrystals [2] using Bragg coherent diffraction imaging [3]. The method demonstrated here does not rely on any a priori shape information and does not involve any ensemble averaging. The implications of this are broad, as it allows determination of the vibrational, mechanical and elastic properties of individual nanocrystals which may be irregularly shaped or contain defects such as stacking faults or dislocations. Additional examples of imaging time-dependent strain in larger crystals using a synchrotron source will also be shown.

In a separate experiment, we demonstrate that in the absence of any temporal resolution, a dynamic system can still be imaged [4]. We will show that dynamics an order of magnitude faster than the intrinsic temporal resolution of the experiment can be imaged. This is achieved by using recent algorithm advances [5] within the lensless imaging technique known as ptychography [6,7]. Potential applications of this are many and include the possibility of imaging fluid flow, magnetic systems or multi-state samples at the nanoscale. 
[1] J. Miao, P. Charalambous, J. Kirz, D. Sayre, Nature 400, 342-344 (1999).

[2] J. N. Clark, et al, Science 341, 56 (2013).

[3] M. A. Pfeifer, et al, Nature 442, 63-66 (2006).

[4] J. N. Clark, et al, Phys. Rev. Lett. 112, 113901 (2014).

[5] P. Thibault \& A. Menzel, Nature 494, 68-71 (2013).

[6] J. M. Rodenburg, et al, Ultramicroscopy 107, 227 (2007).

[7] P. Thibault, et al, Science 321, 379 (2008).

[8] The authors acknowledge funding from Volkswagen Foundation.

A

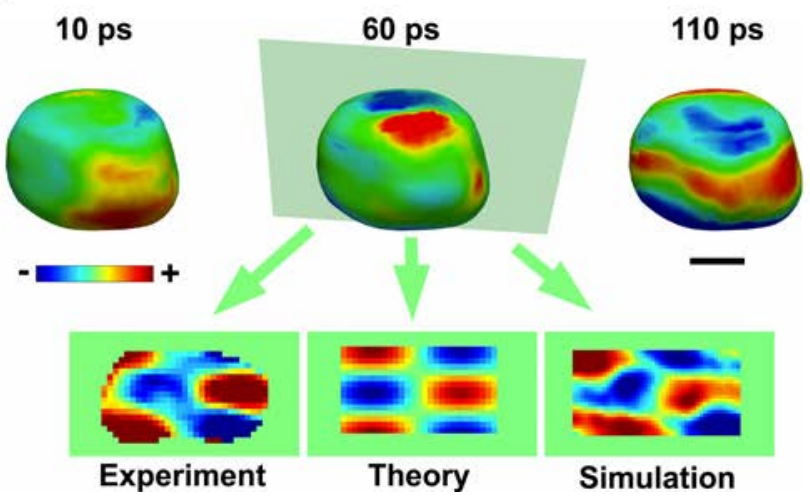

B

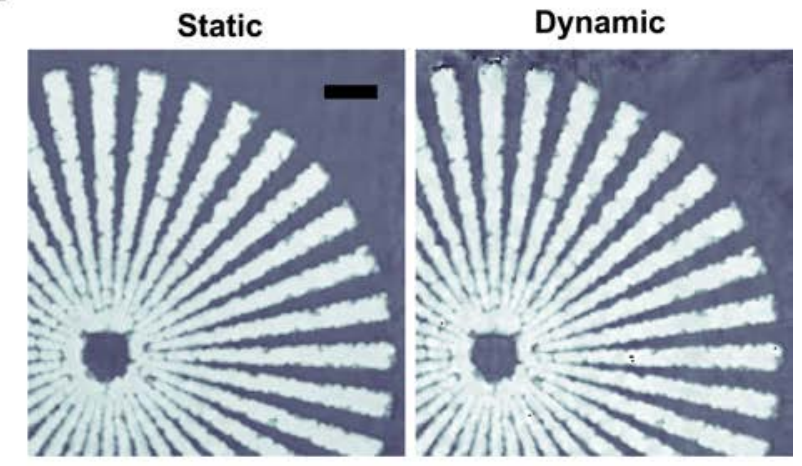

Figure 1. A) Three dimensional images of a nanocrystal showing the deformation due to an optical pump pulse for several different delay times. The experiment is compared to linear elasticity theory (Theory) and molecular dynamics simulation (Simulation). The scale bar is $100 \mathrm{~nm}$. B) A test pattern is imaged when it is stationary (Static) and when it is oscillating (Dynamic) much faster than the temporal resolution of the experiment. The scale bar is $1000 \mathrm{~nm}$. 